apparesls de manocuvre de la station transformalrice prósentent eux-mèmnes une telle complexité, vu la nécessité de tout commander à distance par dos servo-moteurs et de remeltre toute lexplotalion entre les mains d un seul élcctiricien en chef chargé de laire toutes les connexions variables survant les heures du jour et suivant les accidents qui peuvent se produre sur los lignes, qu'il! sserait pratiquemient impossible de subdıviser un paren matéliel entre plusieurs usines fonctionnant en parallèle.

D'ailleurs, au point de vue économique, la concentration peut seule permeltre d'obtentr lénergye ì un prix suffisamment bas pour juslifier la transmission à Paris ; de meme, et récıproque. menl, seul, le transport à Paris peut gager au pont de vue financrer une telle cntrepisse, qui n'exige pas moms de 85 millions de capital d'après noxs calculs, (et mème 100 millıons si lon ajoute différents élémentis de dépense, comme le juge opportun la Commission de la Ville de Parns).

Nos calculs nous ont conduits à installer une puissance totale de 210000 kilowalts dont la moitié sont destinés normalement au transport sur Paris et moitié ì la distribution locale et à l'électrochmme. Dans ces condittuns, la dépense totale d'installation do lusine peut etre considérée comme formée de deux parties ; les 120000 kilowatts destmés a Parls (réserve oomprise) reviennent à environ 330 ou 350 francs par kllowatt installé, les 120000 aulres kilowattis, destmés à des emplois intermittents pour rélectrochumie, reviennent à 100 franos le kilowatt. Ces deux prix se préscntent comme parfartement normaux pour des installatıons hydro-électriques économiques, et franchement inférieurs it ceux de beaucoup d'entreprises de ce genre qui domnent aotuellement de bons résultatis financiers. D'allleurs, le prix du kilowatt utile transporté à Paris, en y comprenant une perte en ligne de 10 it $15 \%$ et les frais d'installation de la ligne, ne ressom pas à plus de 800 franos, prix qui permet, comme on le sait, à l'énergie hydro-électrique de lutter avec avantage contre la vapieur, grice a l'absence de toute dépense de combustible.

Telles sont, en définttive, les causes très complexes, comme on le volt, qui permettent de considérer commle parfatement rationnelle, au poinl de vue technıque et économique, une entreprise qu a pu paraitre, au premer abord, plus ou moins hasardeuse, et de fonder sur elle les mıelleures espéranoes de succès.

\section{LES NOUVELLES LOCOMOTIVES ELEGTRIQUES DU TUNNEL DU SIMPLON}

Depuis l'ouverture de l'exploitation du tunnel du Simplon, en igo6, la traction dans ce tunnel se fatt au moyen de locomotives électriques à courant triphasé, ainsi que nos lecteurs le savent déjà, les premières locomotives ayant été décrites dans La Houille Blanche de juin r 909, en même

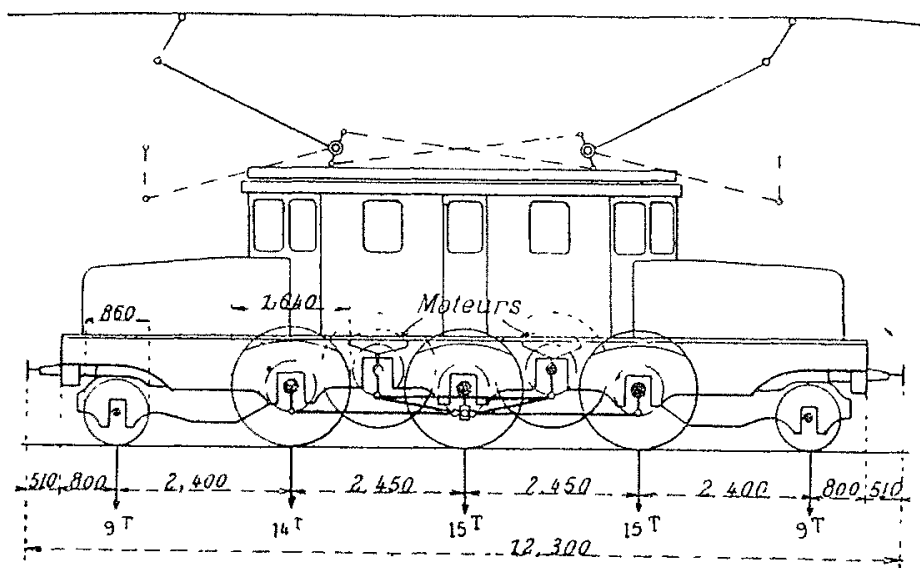

FIG. 1. - Schéma d'une ancienne locomotive.

temps que les installations génératrices hydro-électriques de Brigue et d'Iselle. Depuis igo 8 , le nombre des locomotives ayant été augmenté de deux unités d'un type légèrement différent, nous allons décrire ces nouvelles locomotives, qui, comme les anciennes, sortent des ateliers Brown-Boveri, de Baden (Suisse); mais, au paravant, nous allons rappeler les principales caractéristiques du tunnel du Simplon, ainsi que celles des anciennes locomotives.

La longueur du tunnel du Simplon est de $19770 \mathrm{~m}$., don I 9322 en alignement droit. L'entrée du côté suisse se fait à Brigue, avec une courbe de 350 mètres de rayon, à la cote $685 \mathrm{~m} 33$, et la sortie du côté italien à Iselle, avec une courbe de 400 mètres de rayon, à la cote $633 \mathrm{~m} 60$. La rampe du côté suisse est de 2 pour 1000 sur $9,6 \mathrm{~km}$., et la pente du côté italien de 7 pour Iooo sur 10,2 kilomètres.

Les anciennes locomotives, dont la figure $I$ donne le schéma comparé à celui des nouvelles machines (fig. 2), sont à 3 essieux moteurs, et sont actionnées par deux moteurs triphasés, alimentés sous la tension de 3000 volts, à la fréquence de 16 périodes par seconde. Les manivelles calées sur les arbres des moteurs sont accouplées entre elles all moyen d'une bielle dont le milieu est relié avec l'essieu moteur central, puis avec chacun des autres essieux porteurs au moyen d'une seconde bielle. Un essieu indépendant à l'avant, et un autre à l'arrière, complètent le système roulant. Les moteurs électriques sont connectés de manière à avoir 8 ou ${ }_{1} 6$ pôles, ce qui, avec des roues de ${ }_{1} \mathrm{~m} 64$, correspond à des vitesses de 70 ou de $35 \mathrm{~km}$. à l'heure. La puissance normale est de goo chevaux, et la puissance máxima de I 200 chevaux.

Nouvelles locomotives. - Ces machines possèdent quatre régimes de marche au lieu de deux, ce qui améliore notablement le système de traction électrique par courant électrique, au point de vue de la variabilité de la vitesse. (Cette innovation remarquable est due à $M$. Aichele, ingé. nieur en chef des ateliers Brown-Boveri). En outre, les es. sieux sont réduits au nombre de quatre, et sont tous porteurs, de manière à réaliser le maximum d'adhérence.

Les moteurs sont fixés sur le chassis de la locomotive d'une manière rigide, et actionnent les essieux par un double système de bielles et de manivelles. On remarquera que la bielle triple, qui relie les manivelles des deux moteurs, se déplace parallèlement à elle-même (comme dans les anciennes locomotives d'ailleurs), ce qui supprime l'inconvénient de l'obliquité de la bielle et des trépidations qu'elle en. traine.

Les deux essieux médiants sont 'dépourvus de jeu dans le sens horizontal, mais, par contre, les essieux extrêmes com.

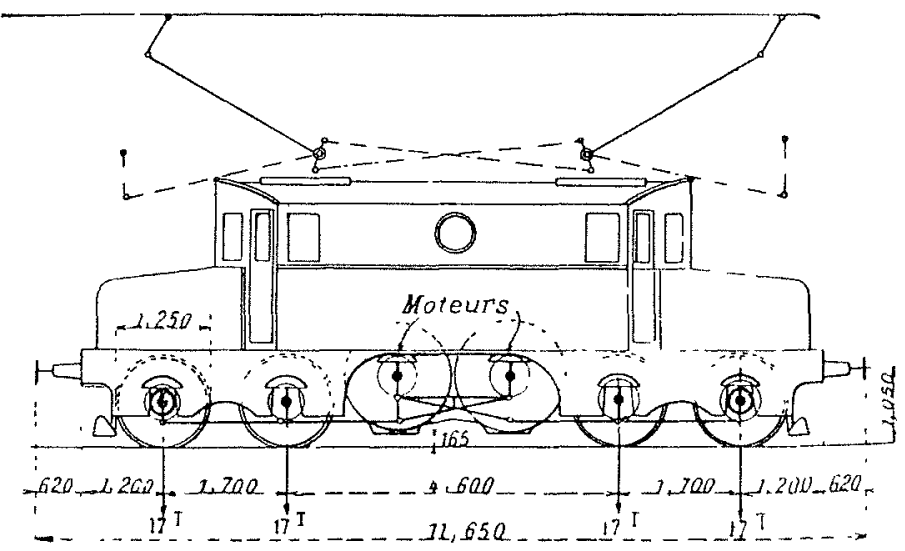

FIG 2, - Ṡchéma d'une nouvelle locomotive.

portent un jeu à la fois latéral et radial pour le passage dans les courbes, le rayon de celles-ci tombant à ı $50 \mathrm{~m}$. aux changements de voie dans les gares. A cet effet, les roues sont calées sur un arbre creux, qui est normalement concentrique à l'axe de l'essieu, et qui supporte le poid de la locomotive par l'intermédiaire d'un truck qui peut tourner légèrement 

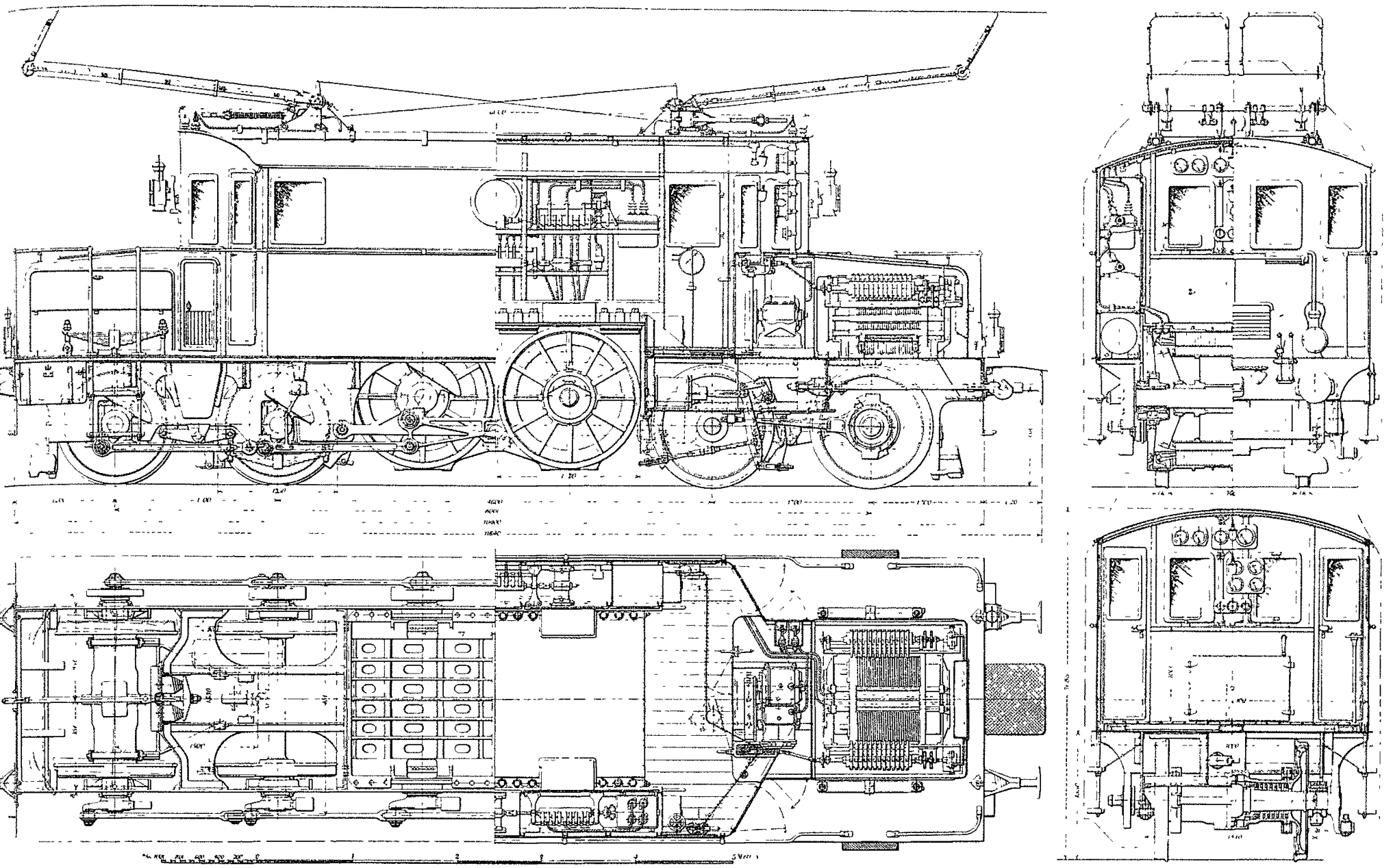

Fig. 3. - Details de construction des nouvelles locomotives du tunnel du Simplon.
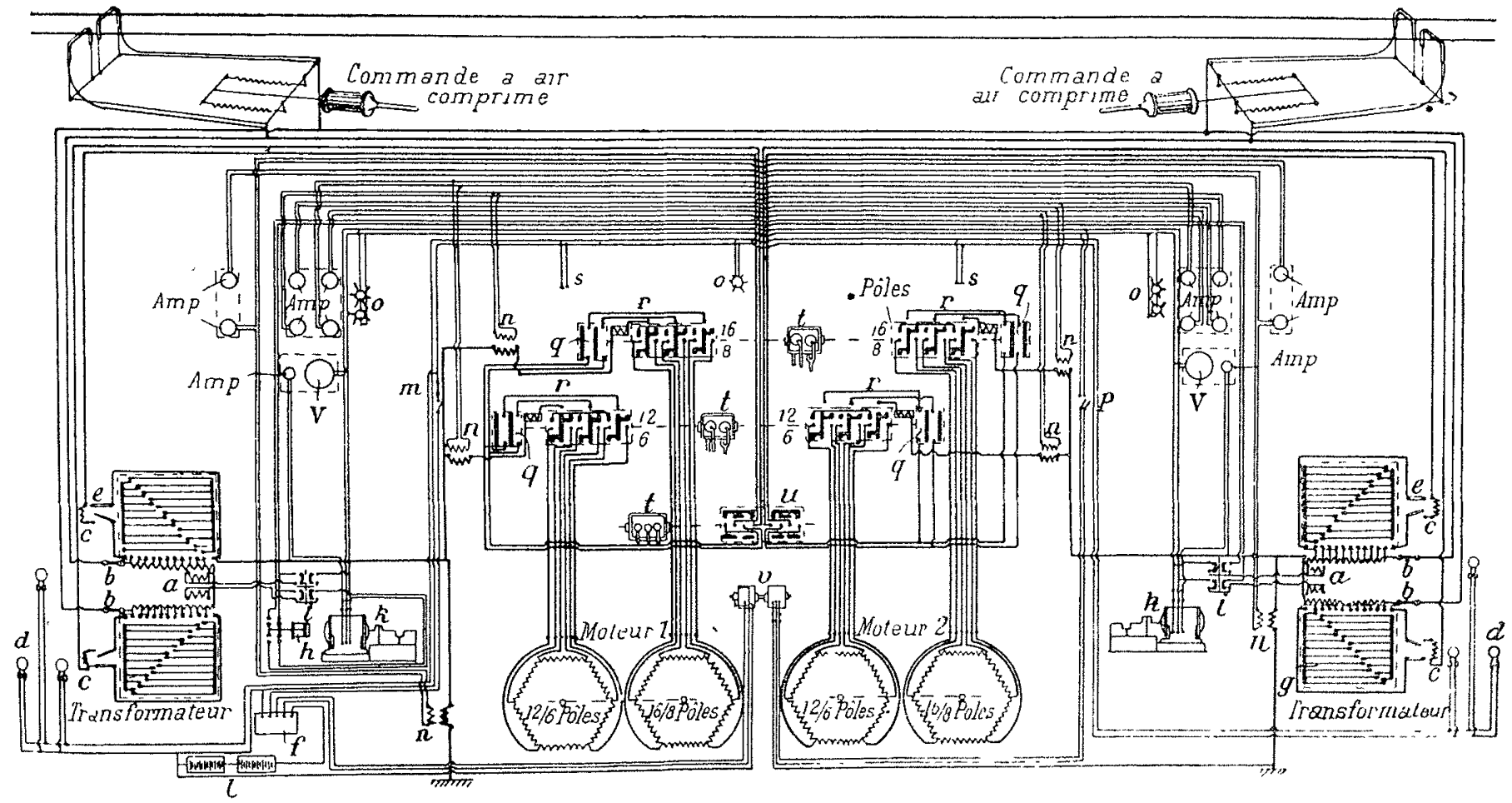

FIG. 4. - Schéma général des connexions électriques des nouvelles locomotives.

IEGEINDE

a, transfolmateur du compresseur $b$, fusibles. éclairage.

c. résistances.

d, lampes des signaux

$\mathfrak{e}$, souffleurs magnétiques. $f$, régulateur automatique pour $o$, contrốleurs des transformateurs $g$, contrôleurs des transformateurs
cipaux. $h$, régulateur automatique de $l$ a piession de l'air.

, commutateurs des compresseurs. $l$, batteric-tampon. m, interrupteur d'eclan age.

$n$, inversen.

o, lampes.

$p$, interropt

$r$, commutateurs de pôles des mo-

$s$, pise de courant des lampes mo- q, disjoncteur haute tension.

t, appatesls de commande à an

comprine

du sens de marche

v, moteur du groupe d'eclarrage.

, amperemetres. 
par rapport à l'axe longitudinal de la locomotive. A l'intérieur de cet arbre creux passe l'axe de l'essieu, qui tourne dans des coussinets portés par le châssis de la locomotive, et qui est actionné par l'extrémité de la bielle correspondante. Un dispositif spécial, représenté en coupe sur la figure 3, provoque l'entraînement de l'arbre creux par l'essieu, tout en permettant un certain jeu.

Le diamètre des roues a été réduit de $\mathrm{I} \mathrm{m}_{4} \mathrm{a} \mathrm{à} \mathrm{I}_{2} 5 \mathrm{o}$, afin de faciliter le passage des courbes, en l'absence de roue simplement porteuse à l'avant.

Les enroulements des inducteurs des moteurs sont hermétiquement fermés ; cette condition très dure, et défavorable à la ventilation, étant imposée par les conditions spéciales de l'exploitation. En effet, pendant l'hiver, les locomotives, qui ont séjourné en gare d'Iselle, où la témpérature descend parfois à $-25^{\circ} \mathrm{C}$, pénètrent rapidement dans le tunnel où l'atmosphère, chargé d'humidité, atteint $+28 \mathrm{Co}$. Il en résulte immédiatement, sur toutes les pièces métalliques encore froides, une forte condensation dont il est absolument indispensable de protéger les enroulements.

Les moteurs, comme nous l'avons dit, comportent 4 régimes, pour 4 vitesses différentes. On a conservé les vitesses de 70 et de $35 \mathrm{~km}$. à l'heure, afin de pouvoir coupler les nouvelles locomotives avec les anciennes, et, pour cela, on a disposé un premier enroulement, dont les connexions déterminent la formation de 6 ou i 2 pôles. Les decix autres
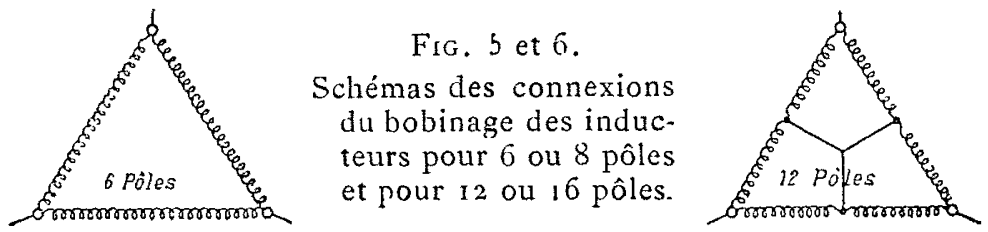

régimes de marche sont obtenus au moyen d'un second enroulement, qui, avec le même système de couplage, formant 8 ou i 6 pôles, correspondent à des vitesses de 52 et 26 kilomètres à l'heure.

La puissance des moteurs, est pour les divers couplages $\begin{array}{lccccc}\text { de } & 850 & 750 & 050 & 550 \mathrm{HP} \\ \text { pour } & 6 & 8 & \text { I } 2 & 16 \text { pôles }\end{array}$

Au démarrage, on met les deux enroulements en parallèle, sur 16 et 12 pôles à la fois, ce qui permet d'obtenir à la fois un couple énergique, égal à la somme des deux couples élémentaires et, en même temps, pour un même couple développé, de répartir l'échauffement produit sur les deux enroulements. Bien entendu, il faut supprimer ce double couplage dès que la vitesse atteint une valeur voisine de 26 kilomètres à l'heure.

Les inducteurs sont constitués par un cylindre creux en tôles feuilletées, de ${ }_{1} \mathrm{~m} 80$ de longueur, et dont les diamètres extérieurs et intérieurs ont respectivement ${ }_{1} \mathrm{~m} 50$ et ${ }_{\mathrm{I}} \mathrm{m}_{\mathrm{I}} \mathrm{O}$. Les induits, ou rotors, sont de simples cages d'écureuil en court circuit, qui fonctionnent indifféremment avec n'importe quel nombre de pôles. C'est, croyons-nous, la première fois qu'on applique ce système à des moteurs de pareille puissance (900 HP), et la chose mérite d'être signalée.

Afin d'éviter d'avoir des courants trop intenses aux démarrages, les courants et les couples y étant à peu près proportionnels au carré de la tension, on a pourvu chaque inducteur, ou stator, d'un double transformateur de démarrage, qui peut réduire la tension au démarrage jusqu'à I ooo volts, puis l'augmenter peu à peu, par crans progressifs de 200 volts, jusqu'à sa valeur maxima, lorsque la vitesse maxima est atteinte. Ces appareils sont logés dans les coffres d'avant et d'arrière des locomotives (voir fig. 3).

Lorsque l'on veut passer d'une vitesse à l'autre, par exemple de $35 \mathrm{~km}$. à l'heure (i 2 pôles) à $52 \mathrm{~km}$.( 8 pôles), on commence par réduire un peu la tension, puis on met l'enroulement à 8 pôles en parallèle avec celui à 12 pôles, on réaugmente le voltage jusqu'à sa valeur normale, puls on met hors-circuit l'enroulement à 12 pôles. (Les commutateurs de pôle sont représentés au bas de la figure 3 , surla demi coupe en plan).

Pour l'arrêt, le mécanicien ramène les transformateurs d̀ I ooo volts, ouvre les commutateurs de pôles en circuit, et ramene l'inverseur de phase au cran d'arrêt. En cas d'ur. gence, il déclanche les disjoncteurs qui coupent immédiatement le courant sur les circuits. Il peut encore abaisser les archets, ceux-ci étant actionnés par l'air comprimé.

L'équipement électrique est fait de telle façon que les connexions d'une locomotive forment deux groupes distincts et symétriques, dont chacun comprend un moteur avec son rédu :teur de tension et tous les appareils de commande et de contrôle correspondants. Toutefois, les archets bipolaires de prise de courant sont connectés à l'ensemble de l'équipement électrique. Les appareils de commande sont installés en double dans les cabines de mancuvre situées à l'avant et à l'arrière du compartiment central, renfermant les moteurs et l'appareillage à haute tension; ils sont asservis entre eux, de manière à éviter toute fausse manœuvre. En cas d'avarie à l'une des cabines, on pourrait continuer le service avec l'autre. Et même, en cas d'accident à l'un des groupes moteurs, on pourrait tant bien que mal continuer le trajet avec l'autre groupe fonctionnant seul.

L'air comprimé nécessaire à la commande des freins età la mancuvre des archets de prise de courant est obtenu au moyen de deux petits compresseurs de $5 \mathrm{HP}$ qui maintien. nent toujours la pression de l'air entre 5 et $7 \mathrm{kgs} \mathrm{par} \mathrm{cm}^{2}$. Les moteurs de ces cumpresseurs sont alimentés par du courant à $\mathrm{l} 00$ volts.

L'éclairage ne s'obtient pas directement au moyendu courant de ligne, pour plus de sécurité, en cas d'interruption subite du courant, mais est assuré par un petit groupe convertisseur qui alimente à la fois les lampes et une petite batterie d'accumulateurs, avec interposition d'un régulateur de voltage système Brown-Boveri.

Les archets bipolaires de prise de courant sont constitués par des cadres métalliques en tubes d'acier, et sont maintenus appuyés contre les fils de ligne par des ressorts. Les frotteurs sont des tubes à section triangulaire, dont les trois faces portent successivement sur les fils de ligne, et durent de 3000 à $5000 \mathrm{~km}$. L'usure des fils de ligne est pour ainsi dire insignifiante : en deux ans, on n'a observé qu'une usur de o, I $2 \mathrm{~mm}$. sur un diamètre de 8 millimètres. Les archets sont reliés aux canalisations de la locomotive par des câbles isolés. Quant aux supports des archets, et à la commande par air comprimé, comme ils sont mis à la terre, leur con tact n'est pas dangereux.

Le tableau ci-joint résume les principales caractéristiques des nouvelles locomotives.

Poids des moteurs.............. $24500 \mathrm{kgs}$

Poids des transformateurs. ........ 6100 "

Poids de l'appareillage $\left({ }^{*}\right) \ldots \ldots \ldots \ldots .4 . \ldots . \ldots$

Poids de la partie mécanique....................

Poids total et poids adhórent...................

$35000 \mathrm{kgs}$

33000 68000

(*) Y compris les deux compresseurs d'air, le groupe convertissel pour l'éclairage et sa batterıe d accumulateurs. 
Poids du matériel électrique par HP effectif.......... Puissance effective en HP

à petite vitesse $(26 \mathrm{~km})$ à grande vitesse $(7 \circ \mathrm{km})$ à petite vitesse $(26 \mathrm{~km})$ à la jante des roues ..... $\ldots$ à grande ritesse $(70 \mathrm{~km})$
uissance effective en $\mathrm{HP}$, à la jante à petite vitesse Puissance effective en HP, à la jante
des roules et par tonne des moteurs, $\left\{\begin{array}{l}\text { à petite vitesse } \\ \text { à grande vitesse }\end{array}\right.$

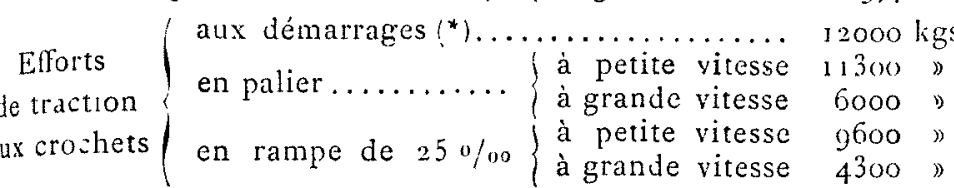

Les puissances et les efforts de traction indiqués sur ce tableau sont bien supérieures à ce qui est nécessaire pour a traction des trains de 300 tonnes à la vitesse de $70 \mathrm{~km}$.; ou de 480 tonnes à la vitesse de $35 \mathrm{~km}$., sur la rampe maxima à $7 \%$ oo du tunnel du Simplon. On les a néanmoins adoptés en prévision de l'accroissement du poids des trains, et de l'extension de la traction électrique entre Iselle et Domodôssola, où se trouve une rampe de $25 \%$ \%

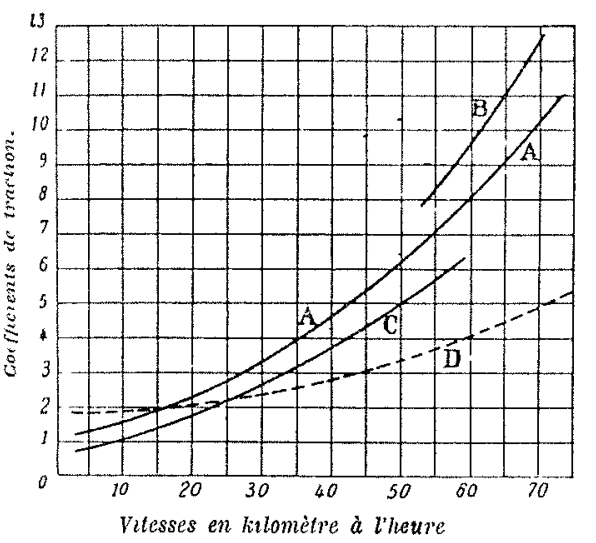

Fig. 7. - Variations du coefficient de traction en fonction des vitesses.
On a tenu compte aussi de ce fait que la résistance de l'air au passage des trains dans le tunnel est vraiment considérable. Il résulte en effet de l'expérience que cette résistance absorbe 700 chevaux à la vitesse de 7 o kilomètres à l'heure. Le train forme piston pour chasser l'air. A ce point de vue, il eût été préférable de ne construire qu'une galerie de grande dimension, comme cela se fait actuellement pour le Loetchsberg, au licu de deux galeries parallèles, comme on l'a fait ici.

Les graphiques de la figure 7 ci-jointe montrent comment varie le cœfficient de traction $\left(^{* *}\right)$ en fonction de la vitesse des trains. Pour les établir, on a fait circuler dans les deux sens un train de 333 tonnes, long de $203 \mathrm{~m}$., soit pendant l'arrêt de la ventilation (courbe A), soit en sens inverse de cette ventilation (courbe B), cette ventilation se faisant avec une vitesse de $3 \mathrm{~m}$. à la seconde (ou de I I $\mathrm{km}$. à l'heure), soit dans le sens de la ventilation (courbe $\mathrm{C}$ ). Rappelons que la section du tunnel est de $23,5 \mathrm{~m}^{2}$, et celle dune locomotive de $9,5 \mathrm{~m}^{2}$. Enfin, à titre de comparaison, on a reporté sur ces graphiques la courbe D, qui correspond aux expériences de traction électrique à très grande vitesse effectuées sur la ligne de Marienfelde à $Z$ ossen $\left(^{* * \star}\right.$ ).

Avant de terminer, signalons que des essais de double traction ont été effectués à diverses reprises en mettant, soit deux machines en tête, soit une en tête et une en queue, et même en accouplant une des anciennes locomotives avec l'une des nouvelles. Ces essais ont montré que la répartition des efforts se faisait d'une facon très satisfaisante, et sans précautions spéciales, de sorte que les craintes conçues à ce sujet étaient exagérées.

J. Collignon, Ingenieur-Electricien.

\footnotetext{
(") Maxımum permis par l'adhérence.

('x) C'est-à-dire l'effort de traction, exprimé en kilogrammes par tonne du train.

$\left(*^{* *}\right)$ Pour la description de ces expériences, voir La Houille Blanche de janvier I904.
}

\section{LOCOMOTIVES ÉLECTRIQUES}

\section{pour la traction des trains de marchandises et des trains à grande vitesse}

Communication faite Ie 7 août 1908 , au Congrès de Clermont-Ferrand de l'Association Francalse pour l'Avancement des Sciences, par M. DE Marchena, ingenicur en chef de la Cie Françalse Thomson-llouston. (Surle)

\section{CONSTITUTION ÉLECTRIQUE DES LOCOMOTIVES}

$S_{1}$ nous passons maintenant à l'examen de la manière dont doivent être électriquement constituées les locomotives, nous abordons une des questions les plus vivement clébattues de l'heure actuelle, une de celles qui ont fait, de la part de praticiens éminents de plusieurs pays, l'objet des controverses les plus étendues et les plus approfondies, sans cependant qu'une conclusion bien nette ait paru jusqu'à préscnt pouvoir être dégagée de ces débats.

Il semble bien à tout le monde qu'au point de vue de l'unification des installations futures, 11 y a1t intérêt primordial à déterminer les grandes lignes et les grandes règles communes auxquelles il conviendrait de les plier ; mais il apparaît également impossible à tous les spécralistes de définır avec certitude ce qu'elles devront être. Les données expérimentales suffisantes manquent encore à l'heure actuclle et aucune controverse théorique ne peut les suppléer.

La difficulté résultant de ce manque de données expérimentales est accrue de ce fait que le choix à farre doit tenir compte d'une quantité d'éléments différents parmi lesquels ne figurent que pour une petite partie les conditions d'établissement des locomotives électriques proprement dites ; enfin, de ce que les raisonnements n'ont pas à porter sur un cas concret bien déterminé dont toutes les données sont bien fixées à l'avance, mais, au contraire, sur un problème vague dont les bases essentielles ne sont prises pour pérsonne.

Néanmoins, nous allons essayer d'examiner cetle question, non pas pour en tirer des conclusions fermes qui pourraient trop facilement être démenties par les faits du lendemain, mais seulement quelques déductions sur les possibilités offertes,

A l'heure actuelle, pour l'électrification des voies ferrées, trois systèmes différents sont principalement envisagés qui ont charcun leurs partisans convaincus et leurs avantages propres indéniables. Ces systèmes sont :

$I^{\circ}$ Le système à courant alternatif triphasé.

$2^{\circ}$ Le système à courant alternatif monophasé

$3^{\circ}$ Le système à courant continu, analogue à celui actuellement en usage courant, mais avec voltage d'alimentation considérablement plus élevé.

Nous allons en passer en revue les caractéristiques essentielles, en essayant de faire la séparation cntre celles de ces aractéritiques qui peuvent se trouver heureusement modifiées dans la suite par les progrès continus de l'électricité, et celles, au contraire, qui, par leur essencc même, sont permanentes et sans modification possible.

Système triphasé. - Nous n'aurions peut-ĉtre pas compris ce système dans l'énumération ci-dessus sans les belles installations réalisées en Italie par MM Ganz ct Cie, et dont le succès a eu trop de retentissement et a marqué un pas trop important dans la traction des chemins de fer pour qu'il fút permis de passer sous silence le système dont elles sont dérivées.

Comme on le sait, les promières expériences de traction par moteurs triphasés sont déjà anciennes. Elles datent déjà d'une douzaine d'années, et sont ducs à MM. Brown-Boveri.

En dehors des lignes à crémaillère ayant un caractère trop spécial, nous pouvons citer notamment la ligne de Burgdorf à Thun, en Suisse, qui constitue un vrai chemin de fer d'intérêt local et qui est $€ n$ exploitation depuis une dizaine d'annécs.

Toutefois, malgré la favcur qui les a accucillies, et les 